$\left.\begin{array}{l}\text { Hardy-Romanujan Journol } \\ \text { Vol. } 6 \text { (1983) } 23-36\end{array}\right\}$

\title{
THE GREATEST SQUARE FREE FACTOR OF A BINARY RECURSIVE SEQUENCE
}

\author{
By T. N. SHOREY
}

8 1. For any sequence of Integers $u_{0}, v_{1}, \ldots, u_{m}, \ldots$ catisfying

$$
u_{m}=r u_{m-1}+8 u_{m-2}, m=2,3 \ldots
$$

where $s$ und $s$ are rational integers vith $x^{2}+4 s \neq 0$, we hove

$$
u_{m}=a \alpha^{m}+b \beta^{m} \cdot m=0,1,2, \ldots
$$

where $\alpha$ and $\beta$ are reots of the polynomial $x^{2}-\varepsilon X-1$ and

$$
a=\frac{u_{0} \beta-u_{1}}{\beta-\alpha}, b=\frac{u_{1}-u_{0} \alpha}{\beta-\alpha} \text {. }
$$

Tho polynomial $\mathrm{X}^{2}-\mathrm{x} \mathrm{X}-\mathrm{g}$ is called the polynomlal mociated to the sequence $\left\{u_{m}\right\}$. The sequesce $\left\{u_{m}\right\}$ is sald to be a non-degenerate binary recuralvo sequence If $a, b, \alpha, \beta$ are non-zero and $\alpha / \beta$ is not a root of unity. For a raticnal integer $x$ with $|\mathbb{| r}|>1$, denote by $P(x)$ the greatest prime factor of $x$ and by $Q(x)$ the grestent square free factor of $x$. If $p_{1}, \ldots, p_{r}$ are all the distinct primes dividing $x$, then $Q(x)=p_{1} \cdots p_{s}$. For non-zero rational integers $x$ and $y$, denote by $[x, y]$ and $(x, y)$, respectively, the leant common multiple and the greatest commos divisor of $x$ and $y$. Further we define $P(1)=P(-1)=1$ and

$$
P\left(\frac{x}{y}\right)=P\left(\frac{x}{(x, y)} \frac{y}{(x, y)}\right)=P\left(\frac{[x, y]}{(x, y)}\right)
$$

and

$$
B\left(\frac{x}{y}\right)=Q\left(\frac{[x, y]}{(x, y)}\right)
$$


Lef $\left\{u_{m}\right\}$ be a non-degenerate blnary recursive sequence glven by (1). Stewast [4] proved that

$$
Q\left(u_{m}\right)>C\left(\frac{m}{\left((\log m)^{2}\right.}\right)^{1 / d}, m>C^{\prime}, \quad \text {. }
$$

where $d=\left[Q(d):^{\prime} Q\right]$ and $C>0, C^{\prime}>0$ are effectively computable numbers depending only on $a$ and $b$. Observé that $d=1$ or 2 . Further, if $|\alpha|>|\beta|$. Stewart $|\psi|$ ] proved that for any $\theta$ with $0<\theta<1$,

$$
\mathrm{Q}\left(\mathrm{u}_{\mathrm{m}}\right)>\mathrm{m}^{\theta}, \mathrm{m}>\mathrm{C}^{\prime \prime},
$$

where $C^{n}>0$ is an effectively computable number depending only on $\theta$ and the sequence $\left\{u_{m}\right\}$. We shall generallie and itrengthen this result as follows :

\section{Theorem 1}

Let $\left\{u_{m}\right\}$ be a non-degenerate binary recursive sequence, There exist effecttvely computable numbers $\mathrm{C}_{1}>0$ and $\mathrm{C}_{2}>0$ depending only on the sequence $\left\{\mathrm{u}_{\mathrm{m}}\right\}$ such that for every $\mathrm{m}>\mathrm{C}_{1}$, we have

$$
\log Q\left(u_{m}\right)>C_{2}(\log m)^{2}(\log \log m)^{-1} .
$$

The improvemest depends on utilising the fact that the contrlbution from small primes in $u_{m}$ is small. Stewart [5] proved theorem 1 for the grestest sqaare free factor of the members of Lucas and Lehmer sequences. Further, for Lucas and Lehmer sequences, Stewart [5] proved that for almost all $\mathrm{m}$

$$
\log Q\left(u_{m}\right)>(\log m)^{2+\log 2-\varepsilon}, \varepsilon>0 \text {. }
$$

Theorem 1 is contalned in the following result. 


\section{Theorem 2}

Let $\left\{u_{m}\right\}$ be a non-degenerate binary recursive sequence. There exist effectively computable numbers $\mathbf{C}_{3}>0$ and $\mathbf{C}_{4}>0$ depending only on the sequence $\left\{\mathrm{u}_{\mathrm{m}}\right\}$ such that for every patr $\mathrm{m}, \mathrm{n}$ with $\mathrm{m}>\mathrm{n}, \mathrm{m}>\mathrm{C}_{3}$ and $\mathrm{u}_{\mathrm{n}} \neq 0$, we have

where

$$
\log Q\left(\Delta_{m, n}^{\prime}\right)>C_{4}(\log m)^{2}(\log \log m)^{-1}
$$

$$
\Delta_{m, n}^{\prime}=\left[u_{m}, u_{n}\right] /\left(u_{m}, u_{n}\right) .
$$

For a non-degenerate binary recurslve sequence $\left\{\mathrm{u}_{\mathrm{m}}\right\}$, observe that the equation $\mathrm{o}_{\mathrm{m}}=0$ Implies that $\mathrm{m}$ is bounded by an effectively computable number depending only ov the sequence $\left\{\mathbf{u}_{\mathbf{m}}\right\}$. We apply theorem 2 with the least integer n ( $D$ is either 0 or 1 ) such that $u_{n} \neq 0$ to obtain theorem 1 . For estimates on $P\left(u_{m}\right)$ and $P\left(\Delta_{m}^{\prime}, n\right)$, wo refer to Stewart [4] and the author [3]. See also the next theorem.

Let $\left\{u_{m}\right\}$ and $\left\{v_{m}\right\}$ be non-degenerate binary recursive sequences whose assoclated polynomials are identically equal. Denote by $\alpha$ and $\beta$ the root of their asscciated polynomlal. Then the sequeace $\left\{u_{m}\right\}$ is given by (1) and (2). Further for $\mathrm{m}=0,1,2, \ldots$, we have

where

$$
v_{m}=a_{1} \alpha^{m}+b_{1} \beta^{m}
$$

$$
a_{1}=\frac{v_{0} \beta-v_{1}}{\beta-\alpha}, b_{1}=\frac{v_{1}-v_{0} \alpha}{\beta-\alpha} .
$$


For $m$ and $n$ with $u_{m} v_{n} \neq 0$, put

$$
\Delta_{m, n}=\left[u_{m}, v_{n}\right] /\left(u_{m}, v_{n}\right)
$$

Then theorem 2 is a particular cese of the following result.

Theorem 3

Let $\mathrm{A}>0$ and $0<\mathrm{K}<(\mathrm{d}+1)^{-1}$ where $\mathrm{d}=[\mathrm{Q}(\mathrm{d}): \mathrm{Q}]$ There exist effectively computable numbers $\mathrm{C}_{5}>0$ and $\mathrm{C}_{6}>0$ depending only on $\mathrm{A}, \mathrm{K}$, the sequences $\left\{\mathrm{u}_{\mathrm{m}}\right\}$ and $\left\{v_{\mathrm{m}}\right\}$ such that for every pair $\mathrm{m}, \mathrm{n}$ with $\mathrm{m}>\mathrm{n}, \mathrm{m}>\mathrm{C}_{5}, \mathrm{v}_{\mathrm{n}} \neq 0$ and

$$
\frac{a \alpha^{m}}{a_{1} \alpha^{n}} \neq \frac{b \beta^{m}}{b_{1} \beta^{n}}
$$

either

$$
\log P\left(\Delta_{m, n}\right)>(\log m)^{A}
$$

or

$$
\sum_{\mathrm{p} \mid \Delta_{\mathrm{m}, \mathrm{n}}^{\mathrm{K}}} 1>\mathrm{c}_{6} \frac{\log \mathrm{m}}{\log \operatorname{lng} \mathrm{m}}
$$

where $\mathrm{p}$ runs through primes

For the proof of tbeorem 2, we may assume $\log P\left(\Delta^{\prime} m, \mathrm{~b}^{\prime}\right)<(\log \mathrm{m})^{2}$. Theo we apply theorem 3 with $\left\{\mathbf{u}_{\mathbf{m}}\right\}=\left\{\boldsymbol{v}_{\mathbf{m}}\right\}, A=2$ and $K=\frac{1}{4}$ Observe that (3) is satisfied, s nce $\alpha / \beta$ is not a root of unity. Now the astertion of theorem 2 follows immedlately.

The proof of theorem 3 depends on the theory of linear forms in logarithms. Let $\alpha_{1}, \ldots, \alpha_{n}$ be non-zero algebraic numb:rs $L$ ot $K$ bo their splitting field over Q.Put $D=[K: Q]$ 
We denote by $A_{1}, \ldots, A_{n}$ upper bounds for the hoights of $\alpha_{1}, \ldots, \alpha_{n}$ respectively, where we assume that $A_{j}>3$ for $1<j<n$. Write

$$
\Omega^{\prime}=\sum_{j=1}^{n-1} \log A_{j}, \Omega=\Omega^{\prime} \log A_{n} .
$$

The proof of theorem 3 depends on the followlog theorem of Baker [1] on linear forms in logarithms.

Theorem A .

There exist effectively computable absolute constants $\mathrm{C}_{7}>0$ and $\mathrm{C}_{8}>0$ such that the inequalities

$$
\begin{aligned}
0<\left|\alpha_{1}{ }^{b_{1}} \ldots \alpha_{n}{ }^{b}-1\right|< & \\
& \quad \exp \left(-\left(C_{7} n D\right){ }^{c_{8} n} \Omega \log \Omega^{\prime} \log B\right)
\end{aligned}
$$

have no solution in rational integers $b_{1} \ldots, b_{n}$ with absolute values at most $\mathrm{B}(>2)$.

We shall also need a p-adic analogue, doe to van dor Poorteo [2], of theorem A.

Theorean B.

Let 8 a a prime ideal of $\mathrm{K}$ lying above a rational prime $\mathrm{p}$. There exist effectively computable absolute constants $\mathrm{C}_{9}>0$ and $\mathrm{C}_{10}>0$ such that the inequalities

$$
\begin{aligned}
& \infty>\operatorname{ord}_{\wp}\left(\alpha_{1}{ }^{b_{1}} \ldots \alpha_{n}{ }^{b_{n}}-1\right)> \\
& \left(C_{9}{ }^{n D}\right)^{C_{10}{ }^{n}} \frac{p^{D}}{\log p} \Omega(\log B)^{2}
\end{aligned}
$$

have no solution in rational integers $\mathrm{b}_{1}, \ldots, \mathrm{b}_{\mathrm{n}}$ with absolute values at most $\mathrm{B}(>2)$. 
8 2. Proof of theorem 3 .

$$
\text { Let } \begin{aligned}
A>0 & \text { and } 0<K<(d+1)^{-1} . \\
\tau & =K(d+1) .
\end{aligned}
$$

Observe that $0<\tau<1$. Let $\left\{u_{m}\right\}$ and $\left\{v_{m}\right\}$ be $\Delta s$ in theroem 3. There is no loss of generality in assumlog that $|\alpha| \geqslant|\beta|$. Then, since $\alpha / \beta$ is not a root of unity, we find that $|\alpha|>1$. For algebraic integer $\varepsilon Q(\alpha)$, denote by [x] the ideal generated by $x$ in the ring of integers of $Q(\alpha)$. There exists a positive rational integer $k$ sach that

$$
\left(\left[\alpha^{2}\right],\left[\beta^{2}\right]\right)=[\mathrm{k}] \text {. }
$$

Put $\alpha_{1}=\alpha^{2} / \mathrm{k}$ and $\beta_{1}=\beta^{2} / \mathrm{k}$. Then the Ideals $\left[\alpha_{1}\right]$ and $\left[\beta_{1}\right]$ are relatively coprime. For $m=0,1,2, \ldots$, notlce that

$$
\begin{aligned}
U_{m} & =k^{-m} u_{2 m}=a \alpha_{1}^{m}+b \beta_{1}^{m}, \\
U_{m}^{\prime} & =k^{-m} u_{2 m+1}=\theta \alpha \alpha_{1}^{m}+b \beta \beta_{1}^{m} \\
v_{m} & =k^{-m} v_{2 m}=a_{1} \alpha_{1}^{m}+b_{1} \beta_{1}^{m}, \\
v_{m}^{\prime} & =k^{-m} v_{2 m+1}=a_{1} \alpha \alpha_{1}^{m}+b_{1} \beta \beta_{1}^{m} .
\end{aligned}
$$

Observe that the sequences $\left\{U_{m}\right\},\left\{U_{m}^{\prime}\right\},\left\{V_{m}\right\}$ and $\left\{\mathbf{v}_{\mathbf{m}}\right\}$ are non-degenerate binary recursive sequences. Bg proving the theorem separately for sequences $\left\{U_{m}\right\}$ and $\left\{V_{m}\right\},\left\{U_{m}\right\}$ and $\left\{V_{m}^{\prime}\right\},\left\{U_{m}^{\prime}\right\}$ and $\left\{V_{m}\right\},\left\{U_{m}^{\prime}\right\}$ and $\left\{\mathbf{V}_{m}^{\prime}\right\}$, there is no lose of generality in assuming that $([\alpha],[\beta])=[1]$.

Denote by $c_{1}, c_{2}, \ldots$ effectively computable positive numbers depending only on $\mathbf{A}, \mathbf{K}$, the sequences $\left\{u_{m}\right\}$ and 
$\left\{v_{m}\right\}$. We may assume that $m>c_{1}$ with $c_{1}$ sufficiently large. Then, since $\left\{u_{m}\right\}$ is non-degenerate, we see that $u_{m} \neq 0$, Let $0<n<m$ satiofy $v_{n} \neq 0$ and suppose lhat (3) is valld. We suppose

$$
\operatorname{leg} P\left(\Delta_{m, n}\right)<(\log m)^{A} .
$$

Let $\pi_{1} \ldots, \pi_{s}$ be all the rational primes satlofying $\pi_{1} \mid \Delta_{m, n}$ and $\pi_{1}>m^{K}$ for $1<1<$. Let $0<\varepsilon<1$. Wo suppose that

$$
s<+\varepsilon(\log m)(\log \log m)^{-1} \text {. }
$$

We ohall arrive at a contradiction for a suitable choice of $\varepsilon$ dependisg only on $\mathbf{A}, \mathbf{K}$ the se quences $\left\{u_{m}\right\}$ and $\left\{v_{m}\right\}$.

We write

$$
\begin{gathered}
B_{2}=\frac{u_{m}}{\left(u_{m}, v_{n}\right)}, B_{2}=\frac{v_{n}}{\left(u_{m}, v_{n}\right)}, \\
\Lambda-\left(u_{m}, v_{D}\right) .
\end{gathered}
$$

Then

$$
\frac{u_{m}}{v_{n}}=\frac{B_{1}}{B_{2}} \text { and }\left(B_{1}, B_{2}\right)=1 .
$$

Further

$$
\Delta_{m, n}= \pm B_{1} B_{2} .
$$

For a prime $p$ divlding $B_{1}$, we see from (7) that

$$
\operatorname{ord}_{p}\left(B_{1}\right)<\operatorname{ord}_{p}\left(a_{m}\right) \text {. }
$$

Let $\gamma$ be a prime ideal in the ring of integers of $Q(\alpha)$ dividing $p$. Then, since the ideals $[\alpha]$ and $[\beta]$ are relatively prime, either $\wp$ does not divide $[\alpha]$ or $\wp$ does not divide $[\beta]$. For simplicliy assume that $\wp$ does not divide $[\alpha]$. Then, by (1), we have 


$$
\begin{aligned}
\operatorname{ord}_{p}\left(u_{m}\right) & <\operatorname{ord}_{\rho}\left(u_{m}\right) \\
& <c_{2}+\operatorname{ord}_{\rho}\left(-\frac{b}{a}\left(\frac{\beta}{\alpha}\right)^{m}-1\right) .
\end{aligned}
$$

Now we apply theorem B with $\mathrm{n}=2, \mathrm{D}=\mathrm{d}, \alpha_{1}=-\mathrm{b} / \mathrm{s}$, $\alpha_{2}=\beta / \alpha, b_{1}=1$ and $b_{2}=m$ to ccnclude that

$$
\begin{aligned}
\operatorname{ord}_{\rho}(- & \left.\frac{b}{a}\left(\frac{\beta}{\alpha}\right)^{m}-1\right) \\
& <c_{3} p^{d}(\log p)^{-1}(\log m)^{+2} .
\end{aligned}
$$

\section{Therefore}

$$
\operatorname{ord}_{p}\left(B_{1}\right)<c_{4} p^{d}(\log p)^{-1}(\log m)^{2} .
$$

Thls Inequalliy follows simllarly whes $\wp 0$ does not divide $[\beta]$. Consequently, by (4),

$$
\sum_{\substack{\mathrm{p} \mid \mathrm{B}_{1} \\ \mathrm{p}<\mathrm{m}}} \text { ord }_{\mathrm{p}}\left(\mathrm{B}_{1}\right) \log \mathrm{p}<\mathrm{c}_{4} \mathrm{~m}^{\tau}(\log \mathrm{m})^{2} .
$$

Similarly

$$
\sum_{\mathrm{pl}_{2} \mathrm{~B}_{2}} \operatorname{ord}\left(\mathrm{B}_{2}\right) \log \mathrm{p}<\mathrm{c}_{5} \mathrm{~m}^{\tau}(\log \mathrm{m})^{2} .
$$

Consequently, by $(9)$, we may write

$$
B_{1}=B_{3} \pi_{1}^{x_{1}} \ldots \pi_{s}^{x_{s}}, B_{2}=B_{4} \pi_{1}^{y_{1}} \ldots \pi_{s}^{y_{s}}
$$

where $x_{1}, \ldots, w_{s}, y_{1}, \ldots, y_{s}$ are non-negative infegers and $B_{3}, B_{4} \varepsilon Z$ with

$$
\log \max \left(\left|\mathrm{B}_{3}\right|,\left|\mathrm{B}_{4}\right|\right)<\mathrm{c}_{6} \mathrm{~m}^{\tau}(\log \mathrm{m})^{2} .
$$


Further we see from (7) that

$$
\log \max \left(\left|B_{1}\right|,\left|B_{2}\right|\right)<c_{7} m
$$

whlch, together with (10), implles tbat

$$
\operatorname{mox}\left(x_{1}, \ldots, x_{8}, y_{1}, \ldots, y_{8}\right)<c_{8} m
$$

with $c_{8}>1$.

Wo bave

$$
\begin{aligned}
& u_{m} a_{1}^{-1} a \alpha^{m-n} v_{n} \\
& =-b_{1} \beta^{n}\left(a_{1}^{-1} a \alpha^{m-n}-b_{1}^{-1} b \beta^{m-n}\right)
\end{aligned}
$$

and, by (7) and (1),

$$
\Lambda B_{1}-a^{\mathbf{m}}=b \beta^{\mathbf{m}}
$$

In view of (3), we see that

$$
u_{m}-a_{1}^{-1} a \alpha^{m-D} v_{n} \neq 0 \text {. }
$$

Put

$$
\begin{aligned}
& T=a_{1}^{-1} a \alpha^{m-n} v_{n} u_{m}^{-1}-1 \\
& r_{1}=a^{-1} \alpha^{-m} \Lambda B_{1}-1,
\end{aligned}
$$

By (15) and (14), notice that

$$
\mathrm{TT}_{1} \neq 0 \text {. }
$$

Further it follows from (8) and (10) that

$$
\begin{aligned}
T_{1} & =a_{1}^{-1} \alpha^{-m} \pi_{1} x_{1} \pi_{8}^{x_{s}}\left(B_{3} \Lambda\right)-1 \\
\text { and } T & =\frac{8}{a_{1}} \alpha^{m-n} \pi_{1}^{z_{1}} \ldots \pi_{s} \frac{B_{3}}{B_{3}}-1
\end{aligned}
$$

where $z_{i}=y_{i}-x_{i}$ for $1<i<8$ Now we oplit the proof of theorem 3 in two cases.

Case I. $|\alpha|>|\beta|$. Divlding both the sides of (13) by $u_{m}$, we bave 


$$
0<\mid \mathbf{T}^{\mathrm{I}}<\mathrm{c}_{9}^{-\mathbf{n}}, \mathrm{c}_{9}>1 .
$$

We apply theorem $i$ with

$n=s+3<\varepsilon(\log m)(\log \log m)^{-1}+3$ by $(6)$,

$D=d<2, \log A_{1}=\log A_{2}=c_{10}, \log A_{3}=\ldots=$

$\log A_{n-1}=(\log m)^{A_{b y}}(5), \log A_{n}=c_{6} m^{\tau}(\operatorname{lcg~m})^{2}$ by (11) and $B=c_{8} m$ by (12) to conclude that

(17) $\quad|\mathrm{T}|>\exp \left(-\mathrm{m}^{\tau+\mathrm{c}_{11} \varepsilon}(\log \mathrm{m})^{5}\right)$.

We shall choose $\varepsilon$ to atisiy

$$
\varepsilon<\left(1-\tau, / 2 c_{11}\right. \text {. }
$$

Put

$$
\tau_{1}=(1+\tau) / 2 \text {. }
$$

Then, since $0<\tau<1$, we find that $\tau<\tau_{1}<1$.

Combining (16), (17) and (18), wo bave

Then

$$
\mathrm{n}<\mathrm{c}_{12} \mathrm{~m}^{\tau_{1}}(\log \mathrm{m})^{5} \text {. }
$$

(19) $\log |\wedge:<\log | v_{n} \mid<c_{13} m^{\tau_{1}}(\log m)^{5}$.

Dividing both the sides of (14) by a $\alpha^{\mathrm{m}}$, we have

(20) $0<\left|\mathrm{T}_{1}\right|<\mathrm{c}_{14}^{-\mathrm{m}}, \mathrm{c}_{14}>1$.

Wo apply theorem $A$ with $n=s+3<\varepsilon(\log m)(\log \log m)^{-1}$ +3 by (6), $D=d<2, \log A_{1}=\log A_{2}=c_{15}$,

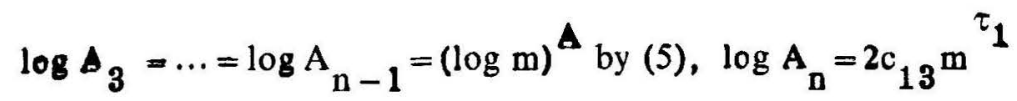
$(\log \mathrm{m})^{5}$ by $(19),(11)$ and $B=c_{8}$ by (12) to conclude that 


$$
\left|\mathbf{T}_{1}\right|>\exp \left(-\mathbf{m}^{\tau_{1}+\mathbf{c}_{16} \boldsymbol{\varepsilon}}(\log \mathrm{m})^{8}\right)
$$

Les

$$
\varepsilon=\min \left(\frac{1-\tau}{2 c_{11}}, \frac{1-\tau_{1}}{2 c_{16}}, \frac{1}{2}\right)
$$

Then (18) is satisfied. Put

$$
\tau_{2}=\left(1+\tau_{1}\right) / 2 \text {. }
$$

Observe that $\tau_{1}<\tau_{2}<1$. Now we sombine (20) a od (21) to conclude that

$$
\mathrm{m} \leqslant c_{17} \mathrm{~m}^{\tau}{ }_{(\operatorname{log~m})^{8}}^{8}
$$

whlch, since $\tau_{2}<1$, implios that $m<c_{18}$. But this is not possible if $\mathrm{c}_{1}>\mathrm{c}_{18}$.

\section{Case II}

$|\alpha|=|\beta|$. Lot $\tau_{1}$ and $\tau_{2}$ be defined as in case $I$. Obse?ve that $\beta$ is not a unit, since $\alpha / \beta$ is not a root of unity. Therefore there exlsts a prime ideal $\delta$ in the rlng of integers of $Q(\alpha)$ such that $\xi /[\beta]$. Further, stnce the tdeals [ $\alpha]$ and $[\beta]$ are relatively coprime, observe that $\odot$ does not divide $[\alpha]$. Consequen!ly ord $\wp_{\rho}\left(u_{m}\right)<c_{19}$. Now, by count!ng the power of prime ideal 5 on both the sides in (13), we have

$$
D<c_{20}+\operatorname{ord}_{f,}\left(u_{\infty}\right)+\operatorname{ord}_{g}(T)<c_{21}+\operatorname{ord}(T) .
$$


We apply theorem $B$ with $\mathrm{p}<\mathrm{c}_{22}$ and the sume parameters as In ease I for obtaining a lower bound for | I | by theorem A. We obtrin

$$
\operatorname{ord}_{\wp}(T)<m^{T+c_{23} \varepsilon}(\log m)^{5} .
$$

We shall choose $\varepsilon$ to astisfy

$$
\varepsilon<\frac{1-\tau}{2 c_{23}} \text {. }
$$

Then

$$
\mathrm{n}<\mathrm{c}_{24} \mathrm{~m}^{\mathrm{T}_{1}}(\log \mathrm{m})^{5}
$$

wbich implles that

$$
\log |\wedge|<c_{25} m^{m_{1}}(\log m)^{5} .
$$

Counting the power of $p$ im ideal $\wp$ on both the sides In (14), we obtain

$$
m<c_{26}+\operatorname{ord}_{\wp}\left(T_{1}\right) .
$$

We apply theorem B with $p<c_{22}, \log A_{n}=c_{25} m^{m_{1}}(\log m)^{5}$ and the same parameters as in case $I$ for obtaining a lower bound for $\left|D_{1}\right|$ by tysorem A We oblaln

Let

$$
\begin{gathered}
\operatorname{ord}_{\rho}\left(T_{1}\right)<m_{1}^{\tau_{1}+o_{27} \varepsilon}(\log m)^{8} . \\
E=\min \left(\frac{1-\tau}{2 c_{23}}, \frac{1-T_{1}}{2 c_{27}}, \frac{1}{2}\right)
\end{gathered}
$$


Then (22) Is sattsfied. We obtain

$$
\mathbf{m}<\mathrm{c}_{28} \mathrm{~m}^{\tau_{2}}(\log \mathbf{m})^{8} .
$$

Conseque ttly $m<c_{29}$ which is not possible if $c_{1}>c_{29}$. Thle completes the proof of theorem 3.

\section{Remarks}

(1) Let $\left\{u_{m}\right\}$ be a non-degenerate blsary recursive sequence. For every palr $m, n$ with $m>n, u_{m} u_{n} \neq 0$ and $Q\left(u_{m}\right)=Q\left(u_{n}\right)$, we have

$$
m-n>c_{30}(\log m)^{2}(\log \log m)^{-1}
$$

whese $c_{30}>0$ Is an e'fectively computable namber dependIng only on the sequence $\left\{u_{m}\right\}$. This follows immediately from theorem 1 and the relation (13) with $a_{1}=a, b_{1}=b$.

(ii) Let $\mathrm{P} \geqslant \mathbf{2}$ and denore by $\mathrm{S}$ the set of all non-zero Integers composed of primes not exceeding $P$. We can apply the algument of proof of theorem 1 to prove that for tvery $x \in S, y \in S$ with $(x, y)=1,|x>| y \mid$ and $\log |x|>e^{e}$,

$$
\log Q(x+y)>c_{31}(\log \log |x|)^{2}(\log \log \log |x|)^{-1}
$$

where $c_{31}>0$ is an effectively computable number depending oniy on $P$. 


\section{References}

1. A. Baker, The theory of linear forms in logarithms, Frenscendence theory: Advances and applications, A. Baker and D.W, Masser ed., Academic Press, London and New York 1977.

2. A. J. van der Poorten, Linear forms in logarithms in the p-adic case, Transcendence theory : Advances and appllcations, A. Baker and Masser ed., Academic Pross, London and New York 1977.

3. T.N. Shorey, Linear forms in members of a binary recursive sequence, Acta. Arith. (to appear).

4. C.L. Stewart, On divisors of terms of Linear recursive sequences, Jour. reine angew Math 333 (1982), 12-31.

5. C.L. Stewart, On divisors of Fermat, Fibonacci, Lucas and Lchmer numbers III, Jour. Lond on Mata. Soc. (to appear).

School of Mathematics

Tata Institute of Fundamental Research

Homi Bhabha Road

Bombay 400005

India. 